\title{
Leveraging copper import by yersiniabactin siderophore system for targeted PET imaging of bacteria
}

\author{
Nabil A. Siddiqui,, ${ }^{1}$ Hailey A. Houson, ${ }^{2}$ Nitin S. Kamble, ${ }^{1}$ Jose R. Blanco, ${ }^{2}$ Robert E. O'Donnell, ${ }^{3}$ \\ Daniel J. Hassett, ${ }^{4}$ Suzanne E. Lapi, ${ }^{2}$ and Nalinikanth Kotagiri ${ }^{1}$ \\ 'Division of Pharmaceutical Sciences, James L. Winkle College of Pharmacy, University of Cincinnati, Cincinnati, Ohio, \\ USA. 'Division of Advanced Medical Imaging Research, Department of Radiology and Chemistry, University of Alabama \\ at Birmingham, Birmingham, Alabama, USA. ${ }^{3}$ Department of Internal Medicine, Heart, Lung and Vascular Institute, \\ and ${ }^{4}$ Department of Molecular Genetics, Biochemistry and Microbiology, College of Medicine, University of Cincinnati, \\ Cincinnati, Ohio, USA.
}

\begin{abstract}
There is an emerging need for accurate and rapid identification of bacteria in the human body to achieve diverse biomedical objectives. Copper homeostasis is vital for the survival of bacterial species owing to the roles of the metal as a nutrient, respiratory enzyme cofactor, and a toxin. Here, we report the development of a copper-64-labeled bacterial metal chelator, yersiniabactin, to exploit a highly conserved metal acquisition pathway for noninvasive and selective imaging of bacteria. Compared with traditional techniques used to manufacture probes, our strategy simplifies the process considerably by combining the function of metal attachment and cell recognition to the same molecule. We demonstrate, for the first time to our knowledge, how a copper-64 PET probe can be used to identify specific bacterial populations, monitor antibiotic treatment outcomes, and track bacteria in diverse niches in vivo.
\end{abstract}

Conflict of interest: NAS, DJH, and NK have filed a patent application with the US Patent and Trademark Office (US patent application no. PCT/ US20/39839) related to this work.

Copyright: (c) 2021, Siddiqui et al. This is an open access article published under the terms of the Creative Commons Attribution 4.0 International License.

Submitted: October 5, 2020

Accepted: April 21, 2021

Published: May 24, 2021

Reference information: /CI Insight. 2021; 6(10):e144880. https://doi.org/10.1172/jici. insight.144880.

\section{Introduction}

Noninvasive imaging of "live" bacteria in the body is in its infancy and a growing, critical, and unmet need, especially during problematic infections caused by multidrug-resistant bacteria. An effective approach should be able to accurately identify the correct pathogen and determine its distribution at the site of infection in order to minimize the indiscriminate use of both narrow- and broad-spectrum antibiotics. Numerous molecular imaging techniques, such as ultrasound, CT, MRI, single-photon emission CT, and PET, have been developed for preclinical and clinical research in the last 3 decades. However, current clinical probes, such as fluorine-18-labeled deoxyglucose $\left({ }^{18} \mathrm{~F}-\mathrm{FDG}\right)$, cannot reliably distinguish bacteria from mammalian cells in vivo (1). Thus, a comprehensive understanding of bacterial physiology and genetics is required to develop probes for targeted imaging. Since bacteria are evolutionarily and phylogenetically distinct from mammalian cells, fundamental differences in metabolism and cellular structures can be leveraged to develop bacteria-specific imaging agents. Some of the recent approaches that have focused on targeting metabolic pathways and proteins unique to bacteria include ${ }^{11} \mathrm{C}$-para-aminobenzoic acid (2), involved exclusively in the bacterial folate pathway; ${ }^{18} \mathrm{~F}$-maltohexaose (3), which specifically targets the maltodextrin transporter in bacteria; and ${ }^{18} \mathrm{~F}$-fluorodeoxysorbitol, a synthetic analog of ${ }^{18} \mathrm{~F}$-FDG that selectively localizes in Gram-negative Enterobacteriaceae (4). However, these radiotracers do not possess the ability to distinguish one genus or species from another. Aside from requiring high specificity and selectivity to the target bacterial population, labeling techniques for generating the reporter probes should be simple, rapid, and inexpensive to qualify as an ideal imaging agent. Current techniques employ a multistep process of labeling metabolites and ligands, which can lead to unnecessary delays and avoidable expenses, especially considering the short half-lives $\left(t_{1 / 2}\right)$ of some radionuclides.

Our study takes advantage of unique natural molecules that serve as both metal chelators and ligands, thus minimizing the labeling protocol to a simple 1-step process. Bacteria have developed sophisticated mechanisms to maintain metal homeostasis within a specified microenvironment. Entire pathways for metal acquisition - comprising (a) de novo synthesis of metal-binding peptide-like molecules known 
as siderophores and (b) dedicated membrane transporters that selectively bind the metal-siderophore complexes - have evolved to precisely regulate this process (5). The central role that these chelators play as chemical ligands in shuttling metals and the unique biology facilitating this transport into bacteria offers a platform that can be harnessed to develop highly versatile and specific contrast agents. In a recent study on the copper homeostasis of pathogenic E. coli UTI89, the siderophore, yersiniabactin (YbT) (Figure 1A), was shown to sequester and transport $\mathrm{Cu}(\mathrm{II})$ from the extracellular environment inside the bacteria (6). The $\mathrm{Cu}(\mathrm{II})-\mathrm{YbT} / \mathrm{Fe}(\mathrm{III})-\mathrm{YbT}$ molar ratio in both human and mouse specimens was consistently greater than 1, suggesting that YbT binds host-derived copper at least as extensively as Fe(III) and demonstrating the physiological relevance of copper binding by YbT (6, 7). Metal-bound YbT (Figure 1B) is first selectively "recognized" by its cognate outer membrane protein receptor, ferric YbT uptake A (FyuA), before the complex is allowed cytoplasmic entry (Figure 1C). We hypothesized that FyuA-expressing bacteria can be targeted by copper-64-labeled $\left({ }^{64} \mathrm{Cu}\right.$-labeled) YbT to facilitate imaging of bacteria with high specificity and sensitivity.

\section{Results}

${ }^{64} \mathrm{Cu}$ readily forms complexes with $\mathrm{YbT}$ with high affinity and stability. To optimize the 1-step radiolabeling process, we labeled YbT with 4 different transition radiometals $-{ }^{55} \mathrm{Co},{ }^{64} \mathrm{Cu},{ }^{68} \mathrm{Ga}$, and ${ }^{89} \mathrm{Zr}$ - in various buffered and temperature-controlled conditions. Primary characterization via instant thin-layer chromatography (iTLC) revealed highest complexation of $\mathrm{YbT}$ with ${ }^{64} \mathrm{Cu}$ (Figure 1D). We elected to analyze ${ }^{64} \mathrm{Cu}-\mathrm{YbT}$ complexation in $0.1 \mathrm{M}$ ammonium acetate buffer $\left(\mathrm{pH}\right.$ ) at $37^{\circ} \mathrm{C}$ further via high-performance liquid chromatography (HPLC). The presence of a single well-defined radio peak (Figure 1E) motivated us to choose this buffered media for probe preparation and all animal experiments. Since YbT was added in excess, a strong, sharp peak for the unreacted fraction was also recorded at around the same time by the UV detector at $220 \mathrm{~nm}$ (Figure 1F). For comparison and as a standard, we employed one of the most promising copper tracers used in preclinical and clinical studies, ${ }^{64} \mathrm{Cu}$-diacetyl-bis(N4-methylthiosemicarbazone) $\left({ }^{64} \mathrm{Cu}-\mathrm{ATSM}\right)(8)$. We noticed that ${ }^{64} \mathrm{Cu}$-ATSM exhibited slightly less efficient ${ }^{64} \mathrm{Cu}$ binding compared with ${ }^{64} \mathrm{Cu}-\mathrm{YbT}$ (Supplemental Figure 1A; supplemental material available online with this article; https://doi. org/10.1172/jci.insight.144880DS1).

To examine the stability of ${ }^{64} \mathrm{Cu}-\mathrm{YbT}$ in vitro, we incubated the probe in mouse serum for $0,0.5,1$, 2 , and 4 hours. We used ethanol/ammonium acetate (50:50) as the extraction solvent and confirmed the presence of at least $60 \%$ of intact ${ }^{64} \mathrm{Cu}$-YbT 4 hours after the initial incubation (Figure $1 \mathrm{G}$ and Supplemental Figure 1B).

${ }^{64} \mathrm{Cu}$-YbT specifically identifies FyuA-expressing bacteria in vivo. Although FyuA-mediated import of ${ }^{64} \mathrm{Cu}-\mathrm{YbT}$ in $E$. coli UTI89 has previously been demonstrated in vitro (6), we sought to validate this phenomenon in vivo. To understand the general biodistribution of ${ }^{64} \mathrm{Cu}-\mathrm{YbT}$, we administered ${ }^{64} \mathrm{Cu}-\mathrm{YbT}$ i.v. to naive mice. PET/CT imaging and ex vivo analysis revealed that ${ }^{64} \mathrm{Cu}-\mathrm{YbT}$ accumulated mostly in the liver and kidneys, with moderate uptake in the heart, lung, and the gastrointestinal tract and minimal uptake in brain, bones, and muscles (Supplemental Figure 2, A and B). We next administered ${ }^{64} \mathrm{Cu}-\mathrm{YbT}$ in mouse models with infectious myositis, generated by injecting bacteria into the hind limb muscles. Substantially higher PET signals (Figure 2, A-C) and activity (Supplemental Figure 2, C-E) were observed from muscles infected with FyuA-expressing E. coli UTI89 (6), E. coli Nissle WT, and Klebsiella pneumoniae $(9,10)$ compared with those infected with control bacteria that do not express a functional FyuA transporter protein. Staphylococcus aureus was used as control as it does not possess the $\mathrm{YbT}$ transport machinery. The other control bacteria was a KO mutant of $E$. coli Nissle $(\Delta f y u A)$, which we constructed by gene replacement of $f y u A$ via the phage $\lambda$ red recombinase technique.

After confirming the specificity of ${ }^{64} \mathrm{Cu}-\mathrm{YbT}$ toward FyuA-expressing bacteria, we sought to determine species selectivity of the probe, relative to E. coli UTI89. We selected Pseudomonas aeruginosa, a Gram-negative and multidrug-resistant nosocomial bacterium similar to E. coli UTI89 and noticed uptake of ${ }^{64} \mathrm{Cu}-\mathrm{YbT}$ more predominantly in muscles infected with E. coli UTI89 (Figure 2D). This is consistent with our previous observation in which bacteria, such as $P$. aeruginosa and $S$. aureus, do not uptake the probe due to absence of the FyuA transporter. Furthermore, to determine the correlation between bacterial viability (live vs. dead) and probe uptake, we administered ${ }^{64} \mathrm{Cu}-\mathrm{YbT}$ in mice infected with live and heat-killed $E$. coli UTI89 and observed signals only from muscles harboring live bacteria (Figure 2E). In addition, we performed comparative uptake studies involving a model of sterile inflammation using turpentine and noticed E. coli UTI89 specificity of ${ }^{64} \mathrm{Cu}$-YbT over turpentine-induced inflamed muscles (Supplemental Figure $2 \mathrm{~F}$ ). 
A

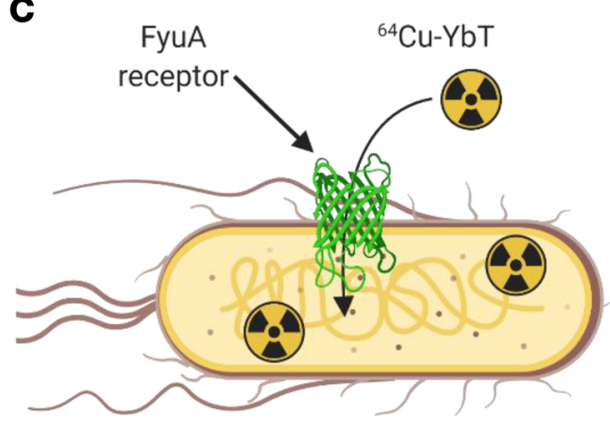

Bacteria

E

Counts

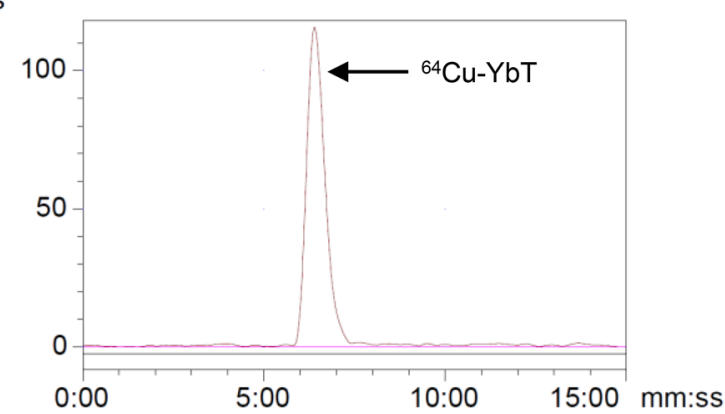

$\mathbf{F}$

mAU

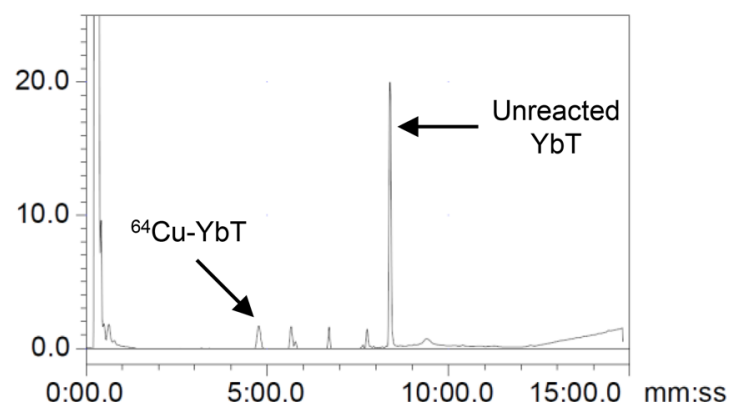

B<smiles>[Y]N1C(C2SCC3CSC(c4ccccc4OC)=[N+]32)CSC1C(C)(C)C(C)(C)C(=O)O</smiles>

$\mathrm{M}-{ }^{55} \mathrm{Co},{ }^{64} \mathrm{Cu},{ }^{68} \mathrm{Ga},{ }^{89} \mathrm{Zr}$

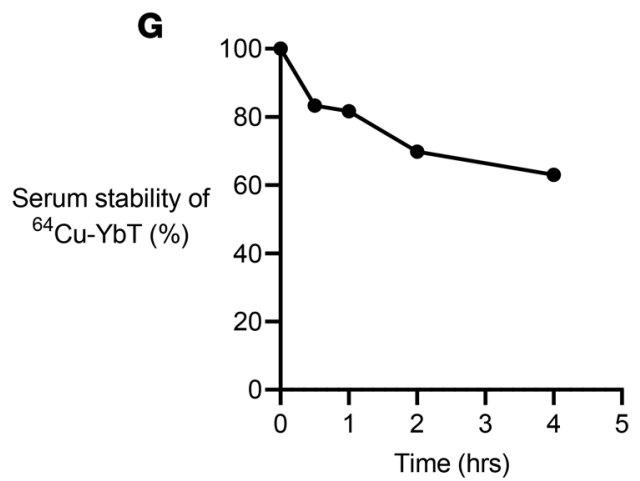

Figure 1. ${ }^{64} \mathrm{Cu}$ readily forms a complex with YbT with high affinity and stability. Chemical structures of (A) Yersiniabactin and (B) metal-YbT complex. (C) Schematic showing selective FyuA-mediated import of ${ }^{64} \mathrm{Cu}-\mathrm{YbT}$ inside bacteria. (D) iTLC characterization of YbT complexation (\%) with ${ }^{55} \mathrm{Co}$, ${ }^{64} \mathrm{Cu},{ }^{68} \mathrm{Ca}$, and ${ }^{89} \mathrm{Zr}$ in various physicochemical conditions. (E) Radio and (F) UV HPLC chromatograms of ${ }^{64} \mathrm{Cu}-\mathrm{YbT}$ prepared at pH 7. (C) Serum stability of ${ }^{64} \mathrm{Cu}-\mathrm{YbT}$ at various time points after incubation. 
A

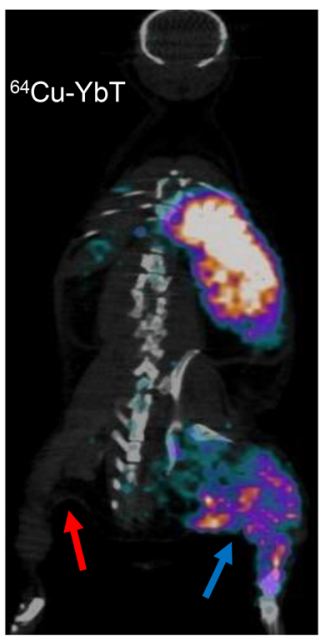

S. aureus vs E. coli UTI89
B

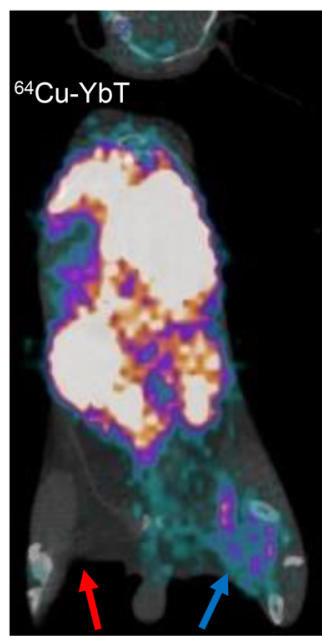

E. coli Nissle vs E. coli Nissle $\triangle$ fyuA
C

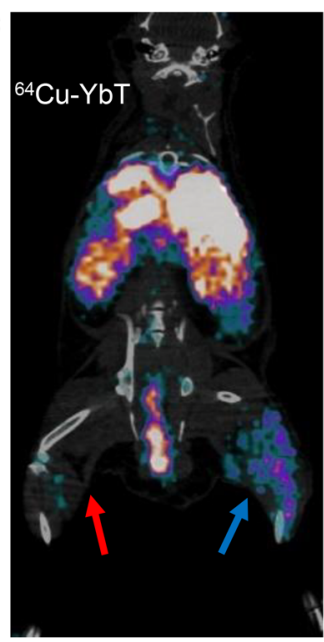

S. aureus vs $K$. pneumoniae

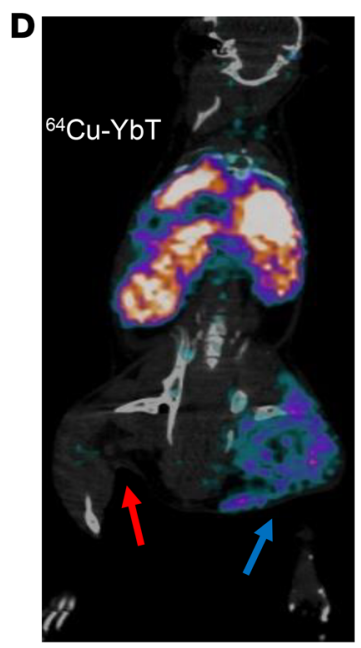

$P$. aeruginosa vs E. coli UTI89

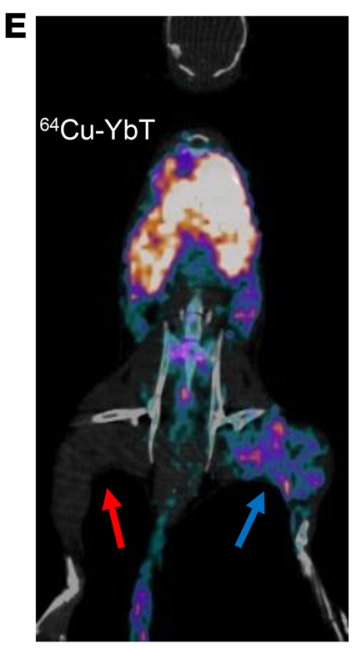

heat-killed vs live (E. coli UTI89)

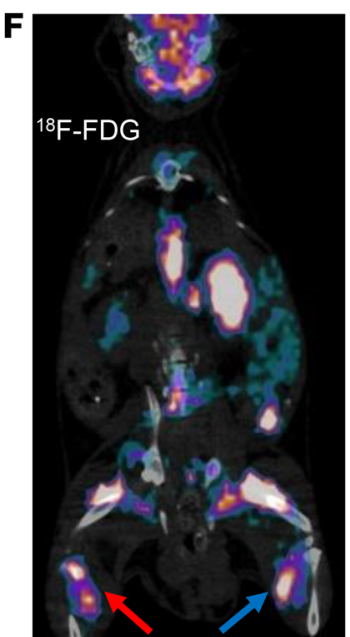

S. aureus vs E. coli UTI89

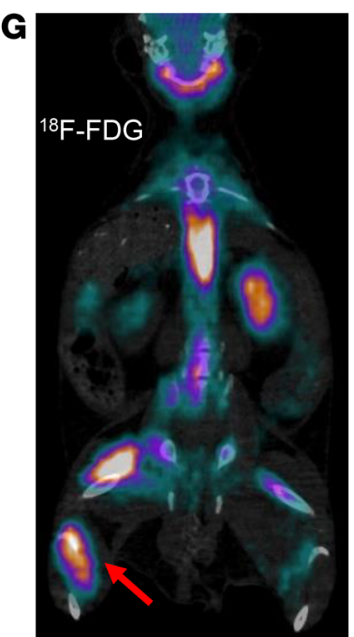

$P$. aeruginosa

Max.

\section{H}

NS

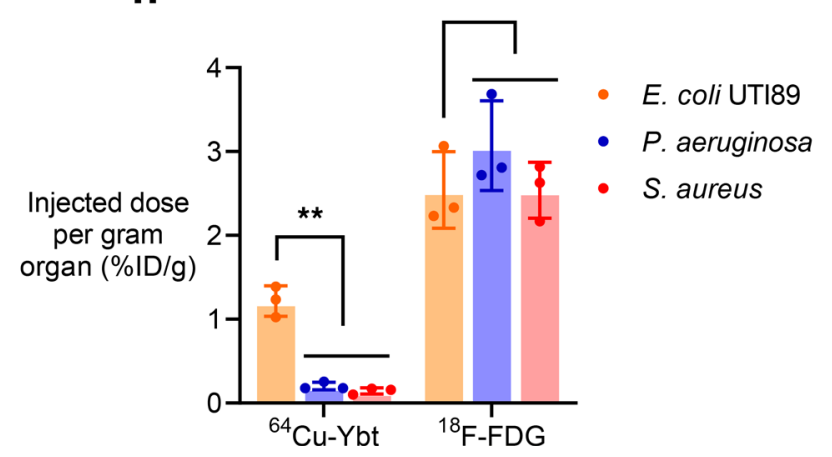

Figure 2. ${ }^{64} \mathrm{Cu}-\mathrm{YbT}$ specifically identifies FyuA-expressing bacteria in vivo. PET/CT images of ${ }^{64} \mathrm{Cu}-\mathrm{YbT} 24$ hours after administration in mice infected with (A) S. aureus and E. coli UTI89, (B) E. coli Nissle WT and E. coli Nissle FyuA-KO mutant ( $\triangle$ fyuA), (C) S. aureus and K. pneumoniae, (D) P. aeruginosa and $E$. coli UTI89, and (E) live and heat-killed E. coli UTI89. PET/CT images of ${ }^{18} \mathrm{~F}-\mathrm{FDC} 2$ hours after administration in mice infected with (F) S. aureus and E. coli UTI89 and (G) P. aeruginosa. (H) Ex vivo biodistribution of ${ }^{64} \mathrm{Cu}-\mathrm{YbT} 24$ hours and ${ }^{18} \mathrm{~F}$-FDG 2 hours after administration in infected mice muscles. Note: arrows indicate sites of bacterial injection. Data are presented as mean \pm SD $(n=3)$ and were analyzed by Greenhouse-Geisser's 1-way ANOVA with Dunnett's multiple-comparison test. ${ }^{* *} P<0.01$. NS, not significant. 
${ }^{64} \mathrm{Cu}$-YbT exhibits higher specificity and comparable sensitivity with clinical PET probes. Any new diagnostic probe must be evaluated against a reliable "gold standard," so we compared the specificity of our probe with the clinically available ${ }^{18} \mathrm{~F}-\mathrm{FDG}$. We noticed that, unlike ${ }^{64} \mathrm{Cu}-\mathrm{YbT},{ }^{18} \mathrm{~F}-\mathrm{FDG}$ accumulated uniformly in muscles harboring pathogenic bacteria, irrespective of their Gram specifications or species differences (Figure 2, F-H). Subsequently, we compared sensitivity of ${ }^{64} \mathrm{Cu}-\mathrm{YbT}$ to ${ }^{18} \mathrm{~F}-\mathrm{FDG}$ by administering the probes in mice infected with $10^{3}, 10^{4}, 10^{5}$, and $10^{6} \mathrm{cfu}$ of 2 different FyuA-expressing bacteria, E. coli UTI89 and $K$. pneumoniae. Figure 3 shows the rapid detection of as little as $10^{4} \mathrm{cfu}$ of both $E$. coli UTI89 and $K$. pneumoniae by ${ }^{64} \mathrm{Cu}-\mathrm{YbT}$ within 2 hours, which is similar to the detection sensitivity of ${ }^{18} \mathrm{~F}-\mathrm{FDG}$. While neither ${ }^{64} \mathrm{Cu}-\mathrm{YbT}$ nor ${ }^{18} \mathrm{FDG}$ was able to sufficiently detect a bacterial load $\leq 10^{4}$ cfu (Figure $3, \mathrm{~A}$ and $\mathrm{B}$ ), their sensitivity was comparable in detecting bacterial loads $>10^{4} \mathrm{cfu}$ (Figure 3, C and D).

Although our in vitro data demonstrated reasonable stability of ${ }^{64} \mathrm{Cu}-\mathrm{YbT}$ in mouse serum (Figure $1 \mathrm{G}$ ), we sought to explore the possibility of "free" ${ }^{64} \mathrm{Cu}$ specifically localizing in muscles infected with FyuAexpressing bacteria by administering an exact dose of unchelated ${ }^{64} \mathrm{Cu}$ in mice infected with E. coli UTI89 and $S$. aureus. We did not observe any significant uptake of "free" ${ }^{64} \mathrm{Cu}$ in either of the muscles (Supplemental Figure 3A), nor was the whole-body biodistribution profile (Supplemental Figure 3B) any different from that of ${ }^{64} \mathrm{Cu}-\mathrm{YbT}$. Next, we compared ${ }^{64} \mathrm{Cu}-\mathrm{YbT}$ with a non-YbT copper-chelated probe, ${ }^{64} \mathrm{Cu}-\mathrm{ATSM}$. In spite of its widespread use in imaging hypoxia in numerous oncologic settings $(11),{ }^{64} \mathrm{Cu}-\mathrm{ATSM}$ is not known to follow any unique copper uptake pathway. Again, we did not observe any significant difference in uptake of the control probe $\left({ }^{64} \mathrm{Cu}-\mathrm{ATSM}\right)$ between the infected muscles (Supplemental Figure 3C), despite the similarity in its general biodistribution to that of ${ }^{64} \mathrm{Cu}-\mathrm{YbT}$ (Supplemental Figure 3D).

${ }^{64} \mathrm{Cu}-\mathrm{YbT}$ can be used to monitor antibiotic treatment efficacy. Clearly, there are several advantages to having an imaging probe capable of performing "real-time" assessment of therapeutic success or failure of antibiotics for the management of seriously ill patients. To demonstrate whether ${ }^{64} \mathrm{Cu}-\mathrm{YbT}$ can potentially be used to monitor therapeutic outcomes, we injected ${ }^{64} \mathrm{Cu}-\mathrm{YbT}$ in mice infected with $E$. coli UTI89 and $K$. pneumoniae to image changes in signal intensity following administration of the broad-spectrum antibiotic, ciprofloxacin. We generated E. coli UTI89 clones with a luciferase reporter to track bacterial growth and burden using bioluminescence imaging (BLI). BLI was able to accurately indicate reduction in bacterial burden in response to antibiotic treatment (Figure $4, \mathrm{~A}$ and $\mathrm{B}$ ). We used ${ }^{64} \mathrm{Cu}-\mathrm{YbT}$ to coregister PET signals with BLI and were able to show a decrease in PET signal corresponding to a proportional decrease in bacterial burden in mice that received 2 doses of ciprofloxacin (Figure 4B), compared with untreated mice (Figure $4 \mathrm{~A})$. When we harvested the thigh muscles from mice, gamma counter analysis revealed significantly lower activity from tissues of mice that received ciprofloxacin compared with the control mice (Figure 4C). These results demonstrate that accurate determination of antibiotic success can be achieved using ${ }^{64} \mathrm{Cu}-\mathrm{YbT}$.

${ }^{64} \mathrm{Cu}-Y b T$ can specifically locate FyuA-expressing bacteria in diverse in vivo environments. Although we successfully demonstrated that ${ }^{64} \mathrm{Cu}-\mathrm{YbT}$ can specifically identify FyuA-expressing bacteria in myositis mouse models, we sought to investigate imaging of these bacteria in a clinically relevant model. We evaluated the efficacy of ${ }^{64} \mathrm{Cu}-\mathrm{YbT}$ in selectively identifying one particular bacteria, K. pneumoniae, in the lung. We developed our mouse models via intranasal administration of $E$. coli Nissle, $K$. pneumoniae, $P$. aeruginosa, and PBS (control). P. aeruginosa, which can also infect the lungs as an opportunistic pathogen, served as the primary negative control. E. coli Nissle served two purposes: (a) it served as a positive control and (b), owing to its nonpathogenic nature, it was used to dismiss any speculation of bacterial pathogenicity playing a role in selective ${ }^{64} \mathrm{Cu}-\mathrm{YbT}$ uptake. PBS was used as the vehicle to administer bacteria in the mice and, hence, served to establish a baseline for the study. After PET/CT imaging with ${ }^{64} \mathrm{Cu}-\mathrm{YbT}$ (Figure 5, A-D), supported by ex vivo biodistribution (Figure $5 \mathrm{E}$ ) of harvested tissues, we observed significantly higher signals from lung and trachea of mice that received $K$. pneumoniae and E. coli Nissle compared with those that received $P$. aeruginos $a$ and PBS. The mottled appearance of the PET signals from the nasal and thoracic cavity indicate and identify clusters of bacteria that dispersed from the point of origin to the trachea, lung, and esophagus. These results illustrate the ability of our probe to track and identify bacteria from the nasal confines to the pulmonary niche with a high degree of both spatial and temporal resolution.

\section{Discussion}

In this study, we performed a comprehensive analysis of multiple radiometal-YbT complexes and successfully identified ${ }^{64} \mathrm{Cu}-\mathrm{YbT}$ as a highly stable $\mathrm{PET}$ probe that can selectively target bacteria with the cognate metal transport protein, FyuA. There is precedent for using siderophores as nuclear imaging contrast agents. 
$\mathbf{A}$
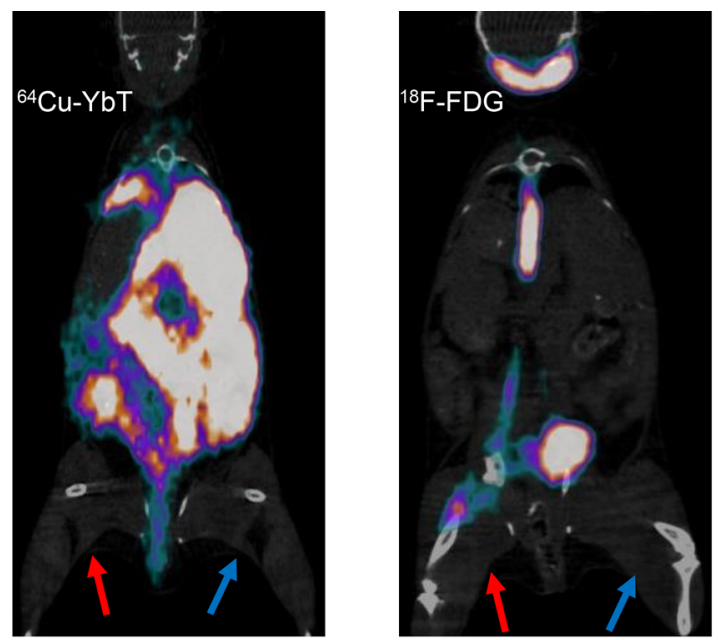

$10^{3} \mathrm{cfu}$

C
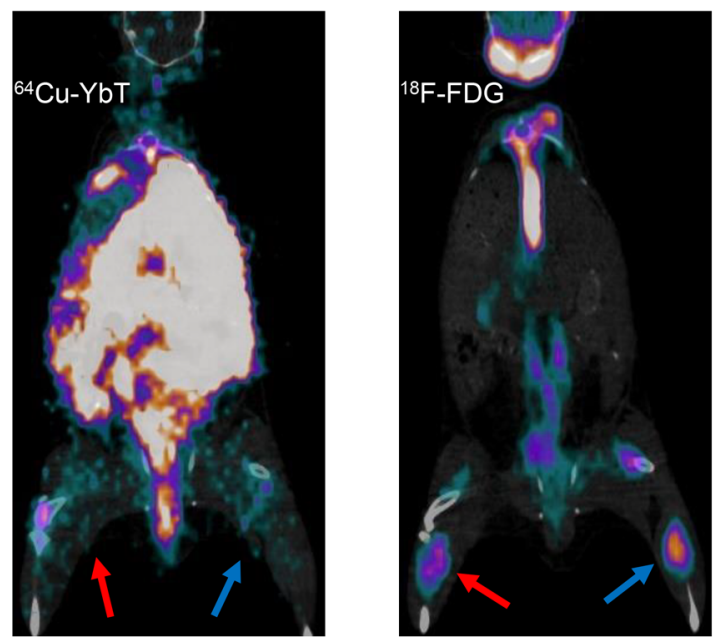

$10^{5} \mathrm{cfu}$
B
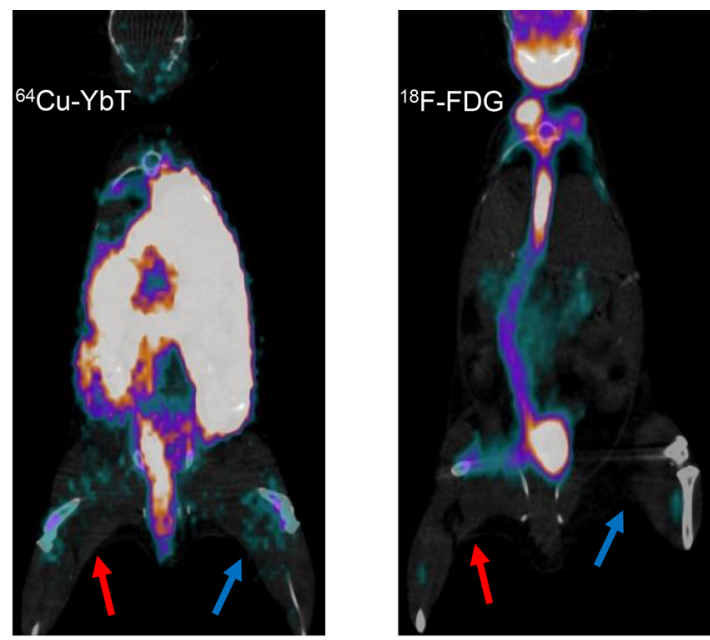

$10^{4} \mathrm{cfu}$
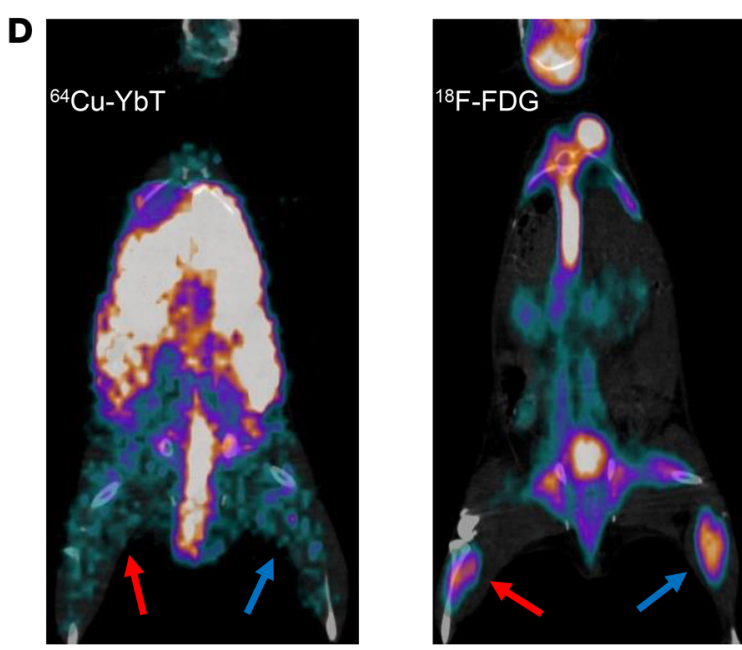

$10^{6} \mathrm{cfu}$

Max.

Figure 3. ${ }^{64} \mathrm{Cu}$-YbT has comparable sensitivity to ${ }^{18} \mathrm{~F}$-FDG. PET/CT images of ${ }^{64} \mathrm{Cu}-\mathrm{YbT}$ and ${ }^{18} \mathrm{~F}$-FDG in mice infected with (A) $10^{3} \mathrm{cfu}$, (B) $10^{4} \mathrm{cfu},(\mathbf{C}) 10^{5} \mathrm{cfu}$, and (D) $10^{6} \mathrm{cfu}$ of bacteria 2 hours after administration of probes. Note: red arrows indicate $K$. pneumoniae and blue arrows indicate $E$. coli UTI89 injection sites.

${ }^{68} \mathrm{Ga}$-labeled siderophores have been used in the past to image fungal infections (12-16). Coordination of ${ }^{89} \mathrm{Zr}$ to desferrioxamine-bound (DFO-bound) vectors, such as antibodies, peptides, and nanoparticles, is a useful strategy for imaging tumor-specific receptors (17). More recently, ${ }^{68} \mathrm{Ga}-\mathrm{DFO}(18)$ and ${ }^{68} \mathrm{Ga}$-pyoverdine (19) probes have been used to image $S$. aureus and $P$. aeruginosa infections in animal models (20). In this study, we have validated that ${ }^{64} \mathrm{Cu}-\mathrm{YbT}$, a metal-siderophore complex specific for certain bacterial populations, can be prepared with high purity and stability under physiological conditions within an hour. The probe can subsequently be used without further purification to selectively complete numerous clinically relevant bacteriological objectives in vivo. The 12.7-hour $t_{1 / 2}$ of ${ }^{64} \mathrm{Cu}$ provides the flexibility to image at both shorter ( 2 hours) and longer ( 24 hours) time scales, allowing optimal clearance of the probe and obtaining images with high contrast (21). Logistically, the $t_{1 / 2}$ also allows the probe to be easily distributed for PET imaging studies at sites remote to the production facility with the loss of approximately $1 t_{1 / 2}$.

We have demonstrated the versatility of ${ }^{64} \mathrm{Cu}-\mathrm{YbT}$ to track pathogenic and probiotic bacteria in distinct in vivo settings; muscle and the lung. E. coli strains UTI89 and Nissle, K. pneumoniae, P. aeruginosa, and $S$. aureus were used for evaluating the probe in muscles. The rationale behind selecting the aforementioned bacteria was to demonstrate the highly selective uptake of ${ }^{64} \mathrm{Cu}$-YbT exclusively in 
A

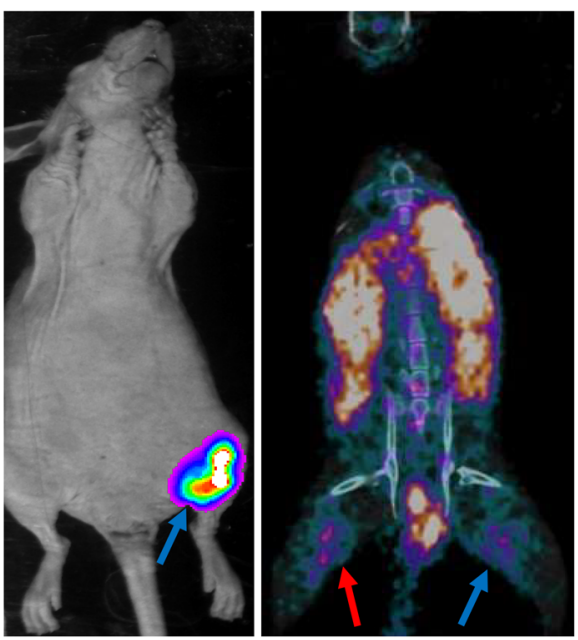

Untreated

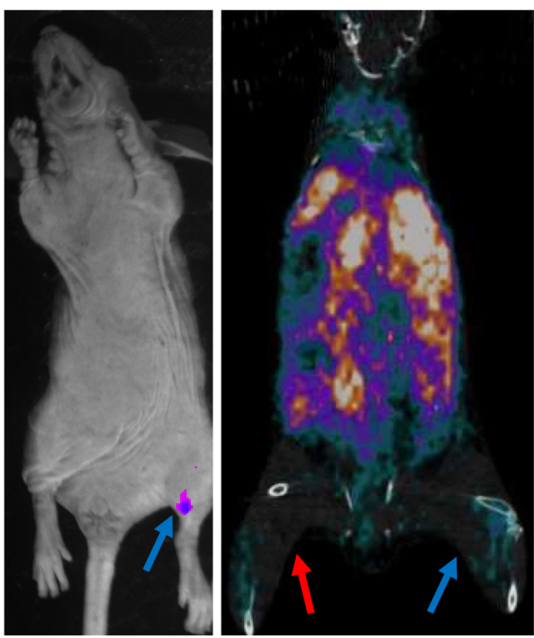

Treated

Max.

Min.

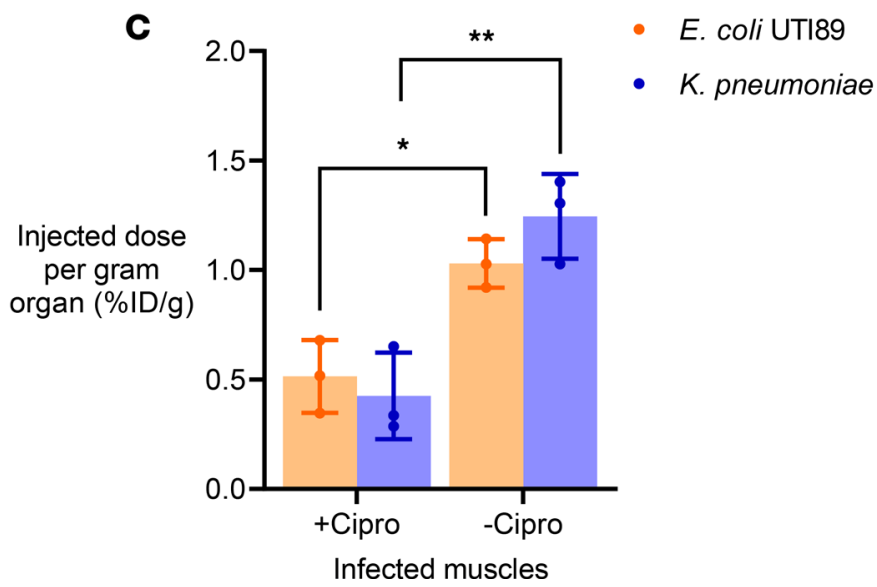

Figure 4. ${ }^{64} \mathrm{Cu}-\mathrm{YbT}$ can be used to monitor antibiotic treatment efficacy. Bioluminescence followed by PET/CT images of (A) mice not treated with ciprofloxacin and (B) ciprofloxacin-treated mice infected with bacteria. (C) Ex vivo biodistribution of ${ }^{64} \mathrm{Cu}-\mathrm{YbT} 24$ hours after administration. Note: red arrows indicate $K$. pneumoniae and blue arrows indicate $E$. coli UTI89 injection sites. Data are presented as mean \pm SD $(n=3)$ and were analyzed by Welch's $t$ test. ${ }^{*} P<0.05,{ }^{* *} P<0.01$.

bacteria expressing the FyuA transporter protein in vivo. In this setting, E. coli UTI89, E. coli Nissle, and $K$. pneumoniae express FyuA, while the rest represent negative controls. For clarity purposes, E. coli UTI89, E. coli Nissle, and K. pneumoniae are Gram-negative bacteria belonging to the Enterobacteriaceae family, of which E. coli UTI89 is a member of the uropathogenic subtype. In contrast, P. aeruginosa is a Gram-negative bacterium belonging to the Pseudomonadaceae family, while $S$. aureus is a Gram-positive bacterium belonging to the Staphylococcaceae family. The central premise of this study was that ${ }^{64} \mathrm{Cu}-\mathrm{YbT}$ has minimal cross-reactivity and has high selectivity across all major levels - family, genus, and serotype - of bacterial class stratification. This is in stark contrast to ${ }^{18} \mathrm{~F}-\mathrm{FDG}$, which is considered a gold standard in clinical infection imaging but is unable to distinguish different types of bacteria. Additionally, we ensured that ${ }^{64} \mathrm{Cu}-\mathrm{YbT}$ was taken up by an equivalent number (Table 1) of live FyuA-expressing bacteria and not nonspecifically in $\triangle f y u A$, dead bacteria, and inflamed regions in the body. In a subsequent study to monitor ciprofloxacin treatment outcome using ${ }^{64} \mathrm{Cu}-\mathrm{YbT}$, we demonstrated that infected muscles with live bacteria "lit up" due to the accumulation of the probe in mice that did not receive the antibiotic. However, no PET signals were observed in mice with identical infected muscles following antibiotic treatment. All this information would be vital in a healthcare setting, where a false positive signal by dead bacteria or inflammation caused by underlying medical conditions could potentially be avoided, preventing misdiagnosis and unnecessary antibiotic usage. 
A

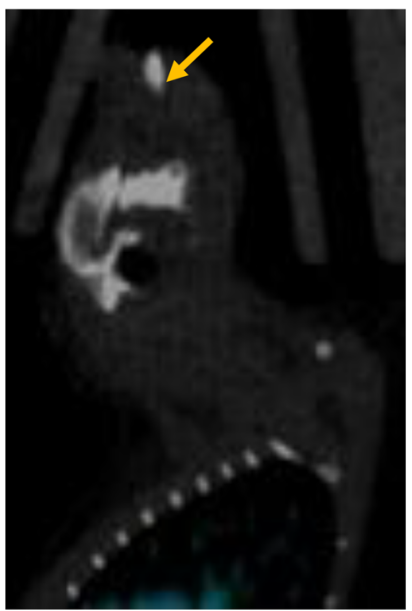

PBS
B

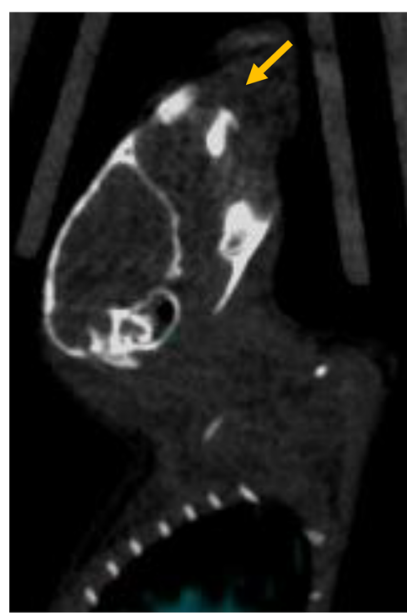

$P$. aeruginosa
C

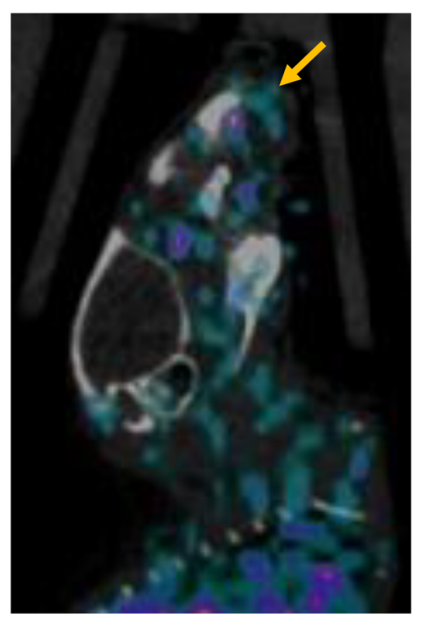

K. pneumoniae
D

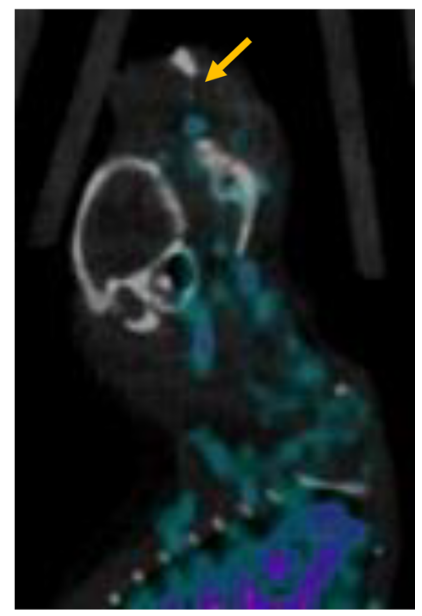

E. coli Nissle

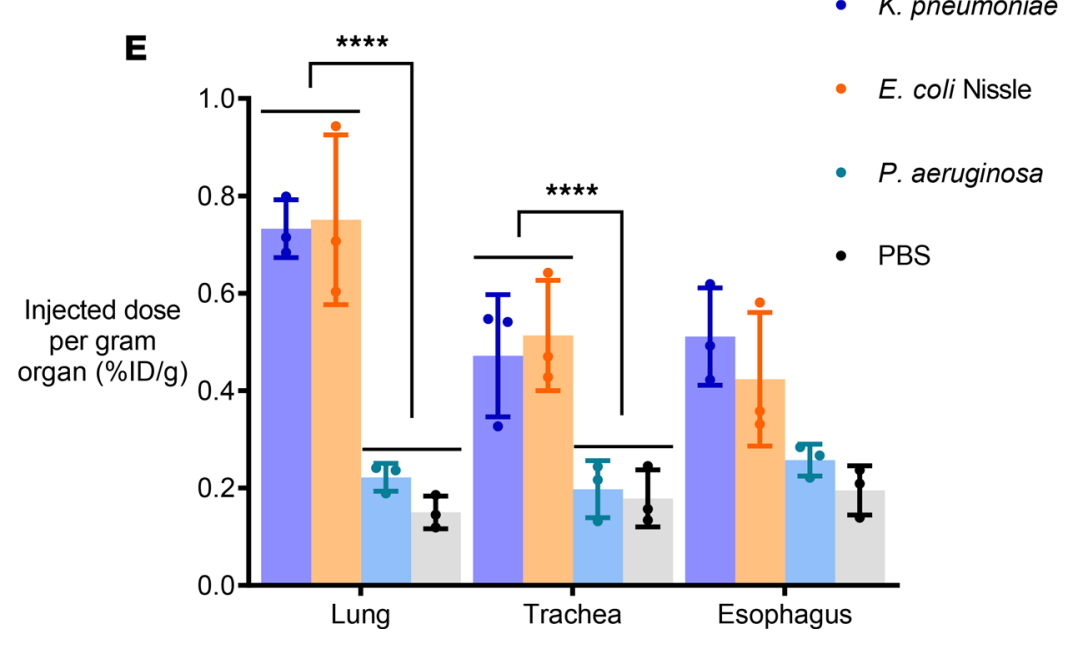

Figure 5. ${ }^{64} \mathrm{Cu}-\mathrm{YbT}$ can specifically locate FyuA-expressing bacteria in diverse in vivo environments. PET/CT images of mice with intranasal administration of (A) PBS, (B) P. aeruginosa, (C) K. pneumoniae, and (D) E. coli Nissle. (E) Ex vivo biodistribution of ${ }^{64}$ Cu-YbT 24 hours after administration. Note: arrows indicate sites of bacterial administration. Data are presented as mean \pm SD $(n=3)$ and were analyzed by 2-way ANOVA with Sidak's multiple comparisons test. ${ }^{* * *} P<0.0001$.

In the past, most PET probes have been developed to target E. coli and S. aureus (22). However, there have not been any significant effort toward the development of a selective imaging modality for the clinically important pathogen, $K$. pneumoniae. Therefore, we were interested in deciphering how rapidly and selectively ${ }^{64} \mathrm{Cu}-\mathrm{YbT}$ could provide a positive signal for FyuA-expressing K. pneumoniae. Hospitals, even in developed countries, run the risk of exposing patients to common pathogens, such as $K$. pneumoniae, $P$. aeruginosa, and methicillin-resistant $S$. aureus, that are known to cause many infections including ventilator-associated pneumonia (23). Particularly, carbapenemase-producing $K$. pneumoniae often prove fatal to patients when the bacteria migrate from the site of infection to the bloodstream, resulting in sepsis. Clinical isolates of $K$. pneumoniae have been shown to modulate immunometabolic pathways to enhance their survival and promote their dissemination in the host (24). Figures 3 and 5 illustrate how our probe can selectively and sensitively depict this phenomenon in vivo.

We have also demonstrated how ${ }^{64} \mathrm{Cu}-\mathrm{YbT}$ can potentially be used to track probiotic bacteria, such as E. coli Nissle, a popular bacterial template for various bioengineering applications (25, 26). Advances in genetic engineering have enabled the exploration of using nonpathogenic bacteria, particularly for cancer therapy, in the last 2 decades $(27,28)$. Selective tracking could allow scientists to better map its location, 
Table 1. Colony-forming units of each type of bacteria recovered 24 hours after intramuscular injection in mice

\begin{tabular}{ccc}
\hline & Bacteria & Colony-forming units $\left(\times \mathbf{1 0}^{\mathbf{6}}\right)$ \\
\hline FyuA $^{+}$ & E. coli Nissle WT & 1.3 \\
& E. coli UTI89 (live) & 0.8 \\
& K. pneumoniae & 0.9 \\
FyuA $^{-}$ & E. coli Nissle $\Delta$ fyuA & 1.3 \\
& E. coli UTI89 (heat killed) & 0 \\
& P. aeruginosa & 1.1 \\
\hline
\end{tabular}

biodistribution, quantity, proliferation, turnover, and fate in the body and, hence, optimize the therapeutic potential of the engineered bacteria and accelerate the translation of bacterial therapies. While numerous optical imaging reporters have been used, bioluminescence and fluorescence have the inherent drawback of limited light penetration, unlike PET imaging, which is depth independent.

In conclusion, we have demonstrated the successful use of YbT as a "dual-role" compound - (a) a ${ }^{64} \mathrm{Cu}$ chelator as well as (b) a targeting ligand for imaging and tracking of pathogenic and probiotic bacteria in vivo. The small probe size ( $\sim 60 \mathrm{Da}$ ), when compared with conventional antibody or peptide-based PET probes, will potentially allow access through the lining of blood vessels to analyze extravascular structures with relative ease. One potential concern regarding the probe could be its specificity to a single bacterial receptor. For example, if the clinical objective is to locate any combination of E. coli UTI89, E. coli Nissle, or $K$. pneumoniae, it would be challenging to delineate the PET signals if both pathogenic and probiotic species are concentrated in or around the same region of the body. Nonetheless, we anticipate further research on the diverse array of natural siderophores as tailored contrast agents for imaging a wide range of pathogenic, probiotic, and engineered bacteria. The same probes can be used across diagnostic platforms for in vitro assays using highly sensitive liquid scintillation and gamma counters as well as in vivo via PET/single-photon emission CT/MRI imaging using appropriate radiometals. Combining siderophores with novel radioisotopes can provide unprecedented insights into unique radiochemistry techniques. For instance, complexation with manganese-52 could facilitate bacterial imaging with MRI or PET/MRI scanners to improve spatial resolution. Such innovations will reveal new and invaluable information on bacteria-host interactions in living systems, which in turn will help design advanced tools and future therapeutic strategies.

\section{Methods}

Chemicals. All reagents were purchased from commercial sources as analytical grade and were used without further purification. $\mathrm{YbT}$ was purchased from EMC Microcollections $\mathrm{GmbH}$. ${ }^{64} \mathrm{Cu}$ was obtained from Mallinckrodt Institute of Radiology, Washington University School of Medicine, St. Louis, Missouri, USA. ${ }^{18}$ F-FDG was purchased from Cardinal Health.

Bacterial strains and culture conditions. E. coli Nissle (Mutaflor), strains E. coli UTI89, P. aeruginosa PAO1, $S$. aureus $\mathrm{Mu} 50$, and a clinical isolate of $K$. pneumoniae (provided in-house) were used in this study. All bacteria were grown in LB agar (BD Difco) plates or LB medium (BD Difco) at $37^{\circ} \mathrm{C}$ as appropriate. FyuA KO was performed in E. coli Nissle using the standard red recombinase method as previously described (Supplemental Figure 4A) (29). All primers (Table 2) were purchased from Integrated DNA Technologies Inc., and the plasmids (Table 3 ) were purchased from Addgene. High-fidelity and colony PCR were performed by using Phusion High Fidelity DNA polymerase (Thermo Scientific) and DreamTaq DNA polymerase (Thermo Scientific), respectively. Success of KO mutagenesis was confirmed by analyzing the colony PCR products of mutant and WT E. coli Nissle via gel electrophoresis (Supplemental Figure 4B). For BLI, E. coli UTI89 was transformed with pAKgfplux1 (Addgene) for constitutive expression of lux genes (30). The positive transformants were selected on Ampicillin plates before use.

Radiometal-YbT complexation - primary characterization. ${ }^{55} \mathrm{Co},{ }^{64} \mathrm{Cu},{ }^{68} \mathrm{Ga}$, and ${ }^{89} \mathrm{Zr}$ were produced at the University of Alabama. Complexation was performed by combining $50 \mu \mathrm{Ci}$ radiometal with $10 \mu \mathrm{g} \mathrm{YbT}$ in $50 \mu \mathrm{L}$ of various buffered media. Samples were incubated at $37^{\circ} \mathrm{C}$ or $90^{\circ} \mathrm{C}$ for 1 hour or 30 minutes, respectively. Binding efficiency was determined via iTLC with $50 \mathrm{mM}$ diethylenetriaminepentaacetic acid as the development buffer. 
Table 2. Primers used in the study

\begin{tabular}{lc}
\hline $\begin{array}{l}\text { Primer } \\
\text { fyuA KO forward }\end{array}$ & Nucleotide sequence \\
fyuA KO reverse & CCATTGTCGGTATTTTTTGGCGTTTCGCCGTCTTACAGGGACTCACAACAGTCTAGGCTGGAGCTGCTTC \\
$\Delta$ fyuA forward & TTAACTAGTGGTGTAAAAGGGATACCTTTTCGGTATCCCTTTTACAATAACATATCAATATCCTCCTTAGTTC \\
CmR forward & CGAGTTTCTGGACCACTGGCCGCTCACCGCC \\
CmR reverse & GAGGCATTTCAGTCAGTTGC \\
$\mathrm{k} 1$ & GCCCAGTCATAGCCGAATAG \\
$\mathrm{k} 2$ & CAGTCATAGCCGAATAGCCT \\
$\mathrm{c} 1$ & CGGTGCCCTGAATCAACTGC \\
$\mathrm{c} 2$ & TTATACGCAAGGCGACAAGG \\
$\mathrm{kt}$ & GATCTTCCGTCACAGGTAGG \\
\end{tabular}

$\mathrm{c} 1$ and $\mathrm{c} 2$ represent chloramphenicol resistance primers. k1, k2, and kt denote different kanamycin resistance primers. CmR, chloramphenicol resistance.

${ }^{64} \mathrm{Cu}$-YbT complexation - secondary characterization. $100 \mu \mathrm{Ci}(10 \mu \mathrm{L})$ of ${ }^{64} \mathrm{Cu}$ (Mallinckrodt Institute of Radiology, Washington University School of Medicine, St. Louis, Missouri, USA) was mixed with $10 \mu \mathrm{g}(10$ $\mu \mathrm{L}$ ) of $\mathrm{YbT}$ in $0.1 \mathrm{M}$ ammonium acetate $(\mathrm{pH}$ 7) to bring the reaction volume to $100 \mu \mathrm{L}$. The mixture was vortexed for $10-15$ seconds before incubating in a thermomixer with $800 \mathrm{rpm}$ agitation at $37^{\circ} \mathrm{C}$ for 1 hour. Labeling efficiency and radiochemical purity were determined using radio-HPLC (Agilent 1260 Infinity II Quaternary System with a Flow-RAM radio-HPLC detector) on a C18 column (Agilent Poroshell 120 EC-C18 column, $3 \times 50 \mathrm{~mm}, 4 \mu \mathrm{m})$. The mobile phase consisted of water (A) and acetonitrile (B) with a gradient of $5 \%-95 \%$ B over 15 min using a flow rate of $1 \mathrm{~mL} / \mathrm{min}$. The retention time of ${ }^{64} \mathrm{Cu}-\mathrm{YbT}$ was 6.35 minutes, and a radiochemical yield of greater than $95 \%$ was achieved and used without further purification.

Serum stability of ${ }^{64} \mathrm{Cu}-\mathrm{YbT}$. In vitro serum stability was conducted by incubating $1 \mu \mathrm{Ci}(10 \mu \mathrm{L})$ of freshly prepared ${ }^{64} \mathrm{Cu}-\mathrm{YbT}$ in $240 \mu \mathrm{L}$ of mouse serum (Thermo Fisher) for $0,0.5,1,2$, and 4 hours. $250 \mu \mathrm{L}$ icecold ethanolic ammonium acetate (50:50) was used to precipitate serum proteins at defined time points by vortexing for $10-15$ seconds followed by centrifugation at $10,000 \mathrm{~g}$ for 5 minutes. The supernatants were analyzed for ${ }^{64} \mathrm{Cu}$-YbT via HPLC using the method described above.

Animal models. Six- to eight-week-old female BALB/cJ mice (The Jackson Laboratory) $(n=3)$ were used for all experiments. Fresh cultures of bacteria were grown to an $\mathrm{OD}_{600}$ of 1.0 at $37^{\circ} \mathrm{C}$ in $\mathrm{LB}$ media while shaking at $200 \mathrm{rpm}$. Bacteria were pelleted at $6000 \mathrm{~g}$ for 2 minutes and washed with PBS 3 times. For muscle infection, $10^{3}$ to $10^{6} \mathrm{cfu}$ of bacteria in PBS were administered intramuscularly; for lung infection, $10^{6} \mathrm{cfu}$ of bacteria were administered intranasally 24 hours before probe administration. To monitor antibiotic treatment efficacy, 2 doses (12 hours apart) of ciprofloxacin (10 mg/kg) were given to mice via oral gavage. This regimen was thought to provide a plasma peak level in the range that was above those required to achieve efficacy after an oral administration of $500 \mathrm{mg}$ ciprofloxacin in humans $(31) .{ }^{64} \mathrm{Cu}-\mathrm{YbT}$ was administered immediately after the second dose of ciprofloxacin.

Imaging and ex vivo biodistribution studies. $100-150 \mu \mathrm{Ci}{ }^{64} \mathrm{Cu}-\mathrm{YbT}$ or ${ }^{18} \mathrm{~F}-\mathrm{FDG}$ in saline $(0.9 \%$ sodium chloride) was injected i.v. in mice. Small-animal PET scan was performed 2 or 24 hours after injection on a $\mu$ PET scanner (Siemens Inveon). Animals were placed in the supine position on the imaging gantry with continued warming for the duration of the scan. CT scan $(80 \mathrm{kVp}, 500 \mu \mathrm{A}$, at 120 projections) was acquired

\section{Table 3. Plasmids used in the study}

$\begin{array}{lc}\text { Plasmid } & \text { Description } \\ \text { pKD } 46 & \text { Expression of } \lambda \text { Red recombinase system for recombineering AmpR } \\ \text { pKD } 3 & \text { Template for overlap PCR and cassette construction CmR } \\ \text { pKD } 4 & \text { Template for overlap PCR and cassette construction KmR }\end{array}$

pKD, plasmid deposited in Addgene (29); AmpR, ampicillin resistance; $\mathrm{CmR}$, chloramphenicol resistance; KmR, kanamycin resistance. 
for anatomical reference overlay with PET image for a 15-minute acquisition with real-time reconstruction. PET images were acquired over an additional 15 minutes and spatial resolution in the entire field of view was determined by ordered subset expectation maximization in 2 dimensions. Histogramming and reconstruction were applied using Siemens Inveon software. Postprocessing was carried out with Inveon Research Workplace, and general analysis was used for contouring volume of interest (VOI). These VOI values were considered active infection volumes and used for further analyses. Bioluminescence images were acquired for 5 minutes using an IVIS Imaging System for quantification of radiance (total flux, photons per second $[\mathrm{p} / \mathrm{s}]$ ) of the bioluminescent signals from the regions of interest. After the imaging studies, the mice were euthanized via carbon dioxide inhalation and cervical dislocation. Organs and tissues of interest were removed and weighed. Residual radioactivity in the samples was measured with a gamma counter, and results are expressed as a percentage of injected dose per gram of organ (\% ID/g). Muscles infected with each type of bacteria were homogenized and serially diluted in fresh LB media, before plating each dilution on an LB agar plate to determine the number of CFU that resulted from the infection.

Statistics. All statistical analyses were performed using GraphPad Prism 9 software. Two-tailed unpaired Student's $t$ test was performed to compare the means between 2 groups, and 1- and 2-way ANOVA were used for 3 or more groups. $P<0.05$ was considered statistically significant.

Study approval. All animal experiments were performed under anesthesia ( $2 \%$ isoflurane), following protocols approved by the University of Cincinnati Biosafety, Radiation Safety, and Animal Care and Use Committees.

\section{Author contributions}

NK conceived the project. NAS and NK designed the study. HAH performed primary characterization of radiometal-YbT complexation. NSK generated E. coli Nissle mutant. NAS performed secondary ${ }^{64} \mathrm{Cu}-\mathrm{YbT}$ characterization, stability, and in vivo experiments. JRB generated radionuclides. NAS and NK analyzed the data and wrote the manuscript with feedback from REO, DJH, and SEL.

\section{Acknowledgments}

We thank Lisa Lemen and Xiangning Wang for technical assistance with the acquisition of all PET/CT images. All schematics were constructed using BioRender.com. This work was supported by the American Heart Association Innovative Project Award (18IPA34170157), the Department of Defense Peer Reviewed Medical Research Program Discovery Award (W81XWH1910125), the National Institute of General Medical Sciences of the NIH (R21GM137321), and NIH Clinical and Translational Science Award program (5UL1TR001425-04).

Address correspondence to: Nalinikanth Kotagiri, 231 Albert Sabin Way, MSB 3005K, Cincinnati, Ohio 45267, USA. Phone: 513.558.6161; Email: kotaginh@ucmail.uc.edu.

1. Ordonez AA, et al. Molecular imaging of bacterial infections: overcoming the barriers to clinical translation. Sci Transl Med. 2019;11(508):eaax8251.

2. Mutch CA, et al. $\left[{ }^{11} \mathrm{C}\right]$ Para-aminobenzoic acid: a positron emission tomography tracer targeting bacteria-specific metabolism. ACS Infect Dis. 2018;4(7):1067-1072.

3. Gowrishankar G, et al. Investigation of 6-[ $\left[{ }^{18} \mathrm{~F}\right]$-fluoromaltose as a novel PET tracer for imaging bacterial infection. PLoS One. 2014;9(9):e107951.

4. Weinstein EA, et al. Imaging Enterobacteriaceae infection in vivo with $18 \mathrm{~F}$-fluorodeoxysorbitol positron emission tomography. Sci Transl Med. 2014;6(259):259ra146.

5. Krewulak KD, Vogel HJ. Structural biology of bacterial iron uptake. Biochim Biophys Acta. 2008;1778(9):1781-1804.

6. Koh EI, et al. Copper import in Escherichia coli by the yersiniabactin metallophore system. Nat Chem Biol. 2017;13(9):1016-1021.

7. Chaturvedi KS, et al. The siderophore yersiniabactin binds copper to protect pathogens during infection. Nat Chem Biol. 2012;8(8):731-736.

8. Liu T, et al. Hypoxia imaging and theranostic potential of $\left[{ }^{64} \mathrm{Cu}\right][\mathrm{Cu}(\mathrm{ATSM})]$ and ionic $\mathrm{Cu}(\mathrm{II})$ salts: a review of current evidence and discussion of the retention mechanisms. EJNMMI Res. 2020;10(1):33.

9. Ohlemacher SI, et al. YbtT is a low-specificity type II thioesterase that maintains production of the metallophore yersiniabactin in pathogenic enterobacteria. J Biol Chem. 2018;293(51):19572-19585.

10. Deriu E, et al. Probiotic bacteria reduce salmonella typhimurium intestinal colonization by competing for iron. Cell Host Microbe. 2013;14(1):26-37.

11. Lapi SE, et al. Evaluation of hypoxia with copper-labeled diacetyl-bis(N-methylthiosemicarbazone). Semin Nucl Med. 2015;45(2):177-185. 
12. Petrik M, et al. 68Ga-siderophores for PET imaging of invasive pulmonary aspergillosis: proof of principle. J Nucl Med. 2010;51(4):639-645

13. Petrik M, et al. In vitro and in vivo evaluation of selected $68 \mathrm{Ga}$-siderophores for infection imaging. Nucl Med Biol. 2012;39(3):361-369

14. Petrik M, et al. Preclinical evaluation of two $68 \mathrm{Ga}$-siderophores as potential radiopharmaceuticals for Aspergillus fumigatus infection imaging. Eur J Nucl Med Mol Imaging. 2012;39(7):1175-1183.

15. Petrik M, et al. 68Ga-triacetylfusarinine $\mathrm{C}$ and $68 \mathrm{Ga}$-ferrioxamine $\mathrm{E}$ for Aspergillus infection imaging: uptake specificity in various microorganisms. Mol Imaging Biol. 2014;16(1):102-108.

16. Petrik $M$, et al. Siderophores for molecular imaging applications. Clin Transl Imaging. 2017;5(1):15-27.

17. Zhang Y, et al. PET tracers based on Zirconium-89. Curr Radiopharm. 2011;4(2):131-139.

18. Petrik M, et al. ${ }^{68} \mathrm{Ga}$-labelled desferrioxamine-B for bacterial infection imaging. Eur J Nucl Med Mol Imaging. 2021;48(2):372-382

19. Petrik M, et al. Imaging of Pseudomonas aeruginosa infection with Ga-68 labelled pyoverdine for positron emission tomography. Sci Rep. 2018;8(1):15698.

20. Petrik M, et al. Siderophore-based molecular imaging of fungal and bacterial infections-current status and future perspectives. J Fungi (Basel). 2020;6(2):73

21. Anderson CJ, Ferdani R. Copper-64 radiopharmaceuticals for PET imaging of cancer: advances in preclinical and clinical research. Cancer Biother Radiopharm. 2009;24(4):379-393.

22. Auletta S, et al. PET radiopharmaceuticals for specific bacteria imaging: a systematic review. J Clin Med. 2019;8(2):197.

23. Kalil AC, et al. Executive summary: management of adults with hospital-acquired and ventilator-associated pneumonia: 2016 Clinical Practice Guidelines by the Infectious Diseases Society of America and the American Thoracic Society. Clin Infect Dis. 2016;63(5):575-582.

24. Codo AC, et al. Inhibition of inflammasome activation by a clinical strain of Klebsiella pneumoniae impairs efferocytosis and leads to bacterial dissemination. Cell Death Dis. 2018;9(12):1182-1182.

25. Kurtz CB, et al. An engineered E. coli Nissle improves hyperammonemia and survival in mice and shows dose-dependent exposure in healthy humans. Sci Transl Med. 2019;11(475):eaau7975

26. Praveschotinunt P, et al. Engineered E. coli Nissle 1917 for the delivery of matrix-tethered therapeutic domains to the gut. Nat Commun. 2019;10(1):5580

27. Chowdhury S, et al. Programmable bacteria induce durable tumor regression and systemic antitumor immunity. Nat Med. 2019;25(7):1057-1063

28. Low KB, et al. Lipid A mutant Salmonella with suppressed virulence and TNFalpha induction retain tumor-targeting in vivo. Nat Biotechnol. 1999;17(1):37-41.

29. Datsenko KA, Wanner BL. One-step inactivation of chromosomal genes in Escherichia coli K-12 using PCR products. Proc Natl Acad Sci U S A. 2000;97(12):6640-6645.

30. Karsi A, Lawrence ML. Broad host range fluorescence and bioluminescence expression vectors for Gram-negative bacteria. Plasmid. 2007;57(3):286-295.

31. Guillard T, et al. Ciprofloxacin treatment failure in a murine model of pyelonephritis due to an AAC(6')-Ib-cr-producing Escherichia coli strain susceptible to ciprofloxacin in vitro. Antimicrob Agents Chemother. 2013;57(12):5830-5835. 